\title{
Effect of Aripiprazole on Cognitive Function and Hyperprolactinemia in Patients with Schizophrenia Treated with Risperidone
}

\author{
Bong Ju Lee ${ }^{1}$, Seung Ju Lee ${ }^{2}$, Min Kyung Kim³ ${ }^{3}$, Jung Goo Lee ${ }^{1,4}$, Sung Woo Park ${ }^{4}$, Gyung Mee Kim, Young Hoon \\ $\mathrm{Kim}^{1,4,5}$ \\ ${ }^{1}$ Department of Psychiatry, Inje University Haeundae Paik Hospital, Inje University College of Medicine, Busan, ${ }^{2}$ Department of Psychiatry, \\ Dongseo Hospital, Masan, ${ }^{3}$ Department of Psychiatry, Dongrae Hospital, ${ }^{4}$ Department of Neuroscience Research, Paik Institute for Clinical \\ Research, Inje University, ${ }^{5}$ FIRST Research Group, Inje University, Busan, Korea
}

\begin{abstract}
Objective: This study aimed to assess the efficacy of aripiprazole for the management of cognitive impairments and hyperprolactinemia in patients with schizophrenia on a stable dose of risperidone.

Methods: Thirty-five subjects stabilized on risperidone (3-6 mg/day) for a minimum of 3 months were enrolled in a double-blind, placebo-controlled phase for 12 weeks and an open-label phase for another 12 weeks. Subjects were randomly assigned to receive $10 \mathrm{mg} /$ day aripiprazole $(n=17)$ or placebo $(n=18)$. Over the following 12 weeks, the the aripiprazole group received a flexible dose of aripiprazole while tapering risperidone. At baseline, week 12, and week 24, subjects were evaluated using the Positive and Negative Syndrome Scale (PANSS), Extrapyramidal Syndrome Rating Scale (ESRS), and standardized neuropsychological assessments. Serum prolactin levels were checked at baseline, week 1, week 2, and week 24.

Results: The mean change in total PANSS and cognitive function test scores between baseline and endpoint were similar in the aripiprazole and placebo groups. Scores on the ESRS and negative subscale of PANSS differed significantly between groups in both phases of the study $(p<0.05)$, indicating a positive effect of aripiprazole. Compared with placebo, aripiprazole significantly reduced mean baseline serum prolactin levels within 1 week $(p=0.015)$.

Conclusion: Adjunctive treatment with and switching to aripiprazole were not associated with improved cognitive function in patients with schizophrenia receiving risperidone; however, aripiprazole treatment decreased negative symptoms and risperidone-induced motor side effects and lowered serum prolactin levels.
\end{abstract}

KEY WORDS: Aripiprazole; Risperidone; Cognition; Prolactin; Schizophrenia.

\section{INTRODUCTION}

Impaired cognition, a core, universal, and disabling feature of schizophrenia, does not have an established treatment. ${ }^{1)}$ Although various approaches have been used to improve cognitive impairment in schizophrenia, ${ }^{2-5)}$ no clear treatment of choice has emerged. Atypical antipsychotics have demonstrated efficacy in treating schizophrenia symptoms and have a low risk of adverse events, such as extrapyramidal symptoms (EPS). Moreover, atypical antipsychotics have been shown to have a beneficial

\footnotetext{
Received: July 3, 2012 / Revised: October 8, 2012

Accepted: February 19, 2013

Address for correspondence: Young Hoon Kim, MD, PhD Department of Psychiatry, Inje University Haeundae Paik Hospital, Inje University School of Medicine and Paik Institute for Clinical Research, FIRST Research Group, Inje University, 875 Haeundaero, Haeundae-gu, Busan 612-862, Korea Tel: +82-51-797-3303, Fax: +82-51-894-6709 E-mail:npkyh@chol.com
}

effect on cognition; however, it is not clear whether this effect is the result of the direct action of the drugs or secondary to the reduction in EPS ${ }^{6,7)}$ However, antipsychotic drug treatment has been associated with the adverse side effect of hyperprolactinemia. ${ }^{89}$ Thus, treatment modalities associated with fewer adverse events of antipsychotic drugs should be considered for the treatment of cognitive impairment in schizophrenia.

Aripiprazole is a dopamine $\mathrm{D}_{2}$ receptor partial agonist with partial agonistic activity at the serotonin-1 $\mathrm{A}\left(5-\mathrm{HT}_{1 \mathrm{~A}}\right)$ receptors and antagonistic activity at the $5-\mathrm{HT}_{2 \mathrm{~A}}$ receptors. ${ }^{10-15)}$ As a result of its unique receptor profile, aripiprazole has a dopamine stabilization effect and has been under investigation as potential cognitive enhancer. ${ }^{16-21)}$ The drug's potentially favorable impact on cognition may be related to the increase in dopamine release in the prefrontal cortex and hippocampus that has been associated with a low dose in animal studies. ${ }^{16)}$ Few studies have in-

(C) This is an Open-Access article distributed under the terms of the Creative Commons Attribution Non-Commercial License (http://creativecommons.org/licenses/by-nc/3.0) which permits unrestricted non-commercial use, distribution, and reproduction in any medium, provided the original work is properly cited. 
vestigated the cognitive-enhancing effects of aripiprazole; most have been open-label studies ${ }^{17-21)}$ and two used a double-blind controlled design. ${ }^{22,23)}$ Moreover, the results of the previous studies were inconsistent. Thus, we investigated the efficacy of aripiprazole using an adjunctive and switching strategy in the same subjects. We hypothesized that switching to aripiprazole following adjunctive therapy would improve cognitive impairment and diminish the risk of side effects associated with risperidone by optimizing $\mathrm{D}_{2}$ and 5- $\mathrm{HT}_{1 \mathrm{~A}}$ receptor activity.

A two-phase study was undertaken to test this hypothesis. The first phase was a 12-week controlled, prospective, double-blind study to investigate the efficacy and safety of aripiprazole versus placebo as an adjunct to a stable regimen of risperidone in patients with schizophrenia. The second phase was a 12-week open-label prospective study in which patients who switched to aripiprazole were compared with those who received a placebo in terms of the stability of their symptoms and the safety of the treatment.

\section{METHODS}

\section{Subjects}

Inpatients from the psychiatric wards of Busan Paik, Dongnae, and Dongseo Hospitals in Korea were enrolled in the present study. Informed consent was obtained from all participants, and the protocol was approved by the Institutional Review Board of Inje University Busan Paik Hospital. All patients met the Diagnostic and Statistical Manual of Mental Disorders-fourth edition, text revision $^{24)}$ diagnostic criteria for schizophrenia and had been stabilized on their current dose of risperidone (3-6 $\mathrm{mg}$ /day) for a minimum of 3 months prior to enrollment in the study. Patients who had any medical problem or took any medication that affected cognitive performance were excluded. Antiparkinsonian drugs, anticholinergics, and benzodiazepines were permitted if the dose did not change during the 24-week study period.

\section{Study Design}

Following baseline evaluations of the severity of psychiatric symptoms and cognitive impairment, 35 subjects were enrolled in a 12-week, double-blind, parallel trial of aripiprazole adjunctive treatment. Upon entry into the double-blind, dual-therapy phase, patients receiving a stable dose of risperidone (3-6 mg/day) were randomly assigned to either adjunctive aripiprazole $(10 \mathrm{mg} /$ day $)$ or placebo in a $1: 1$ ratio for 12 weeks. At the end of the first
12 weeks, subjects in the aripiprazole group received a flexible dose of aripiprazole and tapered risperidone, and the placebo group received their current risperidone dose without placebo for an additional 12 weeks. The Positive and Negative Syndrome Scale (PANSS) ${ }^{25}$ ) was administered at 12 and 24 weeks after the baseline evaluation to assess changes in the severity of psychiatric symptoms. A cognitive assessment battery was used in the same manner to assess changes in several domains of cognitive function after adjunctive treatment with aripiprazole. Subjects were encouraged to report adverse events after administration of aripiprazole, and a simple adverse-events checklist was also used.

\section{Assessment}

The PANSS was used to assess the severity of psychiatric symptoms. Furthermore, scores on the Extrapyramidal Symptom Rating Scale (ESRS) ${ }^{26)}$ were assessed at baseline, week 12, and week 24. Several neuropsychological tests were administered together as a cognitive assessment battery to evaluate changes in several domains of cognitive function. The cognitive assessment battery included the following measures of attention, auditory and visual memory, and executive function: Hopkin's Verbal Learning Test (HVLT); the Rey Visual Learning Test; Digit Span Forward and Backward Test; Digit Symbol Substitution Test, Stroop Test; Trail-Making Tests Part A and B; Verbal Fluency Test (VFT); and Boston Naming Test. The HVLT tests immediate memory span, new learning, susceptibility to interference, and delayed recall. The Trail-Making Test Part A tests visuomotor speed and the ability to set shift. The VFT measures verbal productivity and the intactness of the lexical system. The present study used the category fluency test. Levels of serum prolactin were measured at baseline and at weeks 1, 2, and 24.

Safety assessments included recording of adverse events, physical examination, measurement of vital signs, and laboratory tests at baseline and at weeks 12 and 24 .

\section{Statistical Analysis}

Baseline comparisons between the two groups were performed using the Mann-Whitney U-test for continuous variables and the chi-square test for categorical variables. Comparative efficacy was analyzed using the MannWhitney U-test to examine differences over time (baseline and week 12; baseline and week 24) in clinical and cognitive performance. Changes in serum prolactin levels (baseline, week 1, and week 2; baseline and week 24) were assessed using repeated-measures analysis of variance 


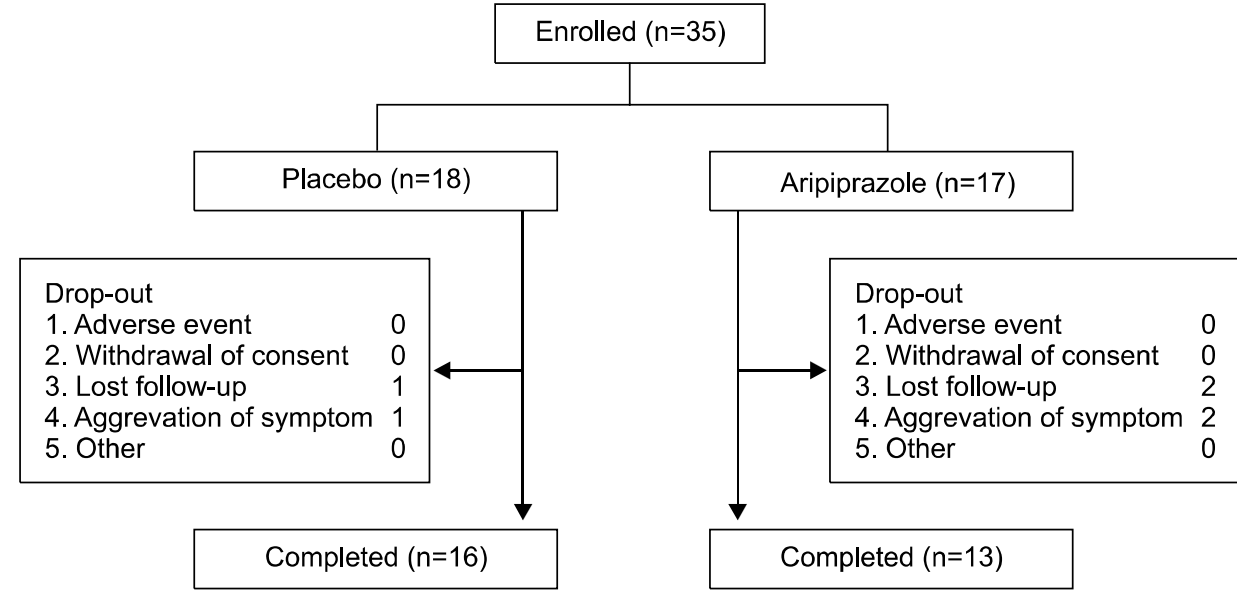

Fig. 1. Patient disposition throughout the study.
(ANOVA) and the Mann-Whitney U-test, respectively. For missing data, the last-observation-carried-forward (LOCF) analysis was used. All statistical tests were conducted using the PASW Statistics version 18.0 for Windows (IBM Co., Armonk, NY, USA), and the results are expressed as mean \pm standard error. All tests were two-tailed, and $p$-values $<0.05$ were deemed statistically significant.

\section{RESULTS}

\section{Demographics}

The 35 subjects enrolled in the study and were randomly assigned to the aripiprazole $(n=17)$ or placebo $(n=18)$ group. Of these, 29 completed the entire protocol and experienced no significant adverse events. Reasons for discontinuing the study were loss to follow-up (two patients in the aripiprazole and one patient in the placebo group) and aggravation of symptoms (two patients in the aripiprazole and one patient in the placebo group; Fig. 1). Patient demographic and clinical data are shown in Table 1. No significant differences in demographic or clinical characteristics were found between aripiprazole and placebo groups. The mean dose of aripiprazole in the aripiprazole group at week 24 was $12.35 \mathrm{mg}$ /day.

\section{Effect of Adjunctive Treatment and Switching to Aripiprazole on Impaired Cognitive Function}

We found no statistically significant between-group differences in the cognitive function tests between baseline and week 12 and baseline and week 24 (Table 2).

\section{Effect of Adjunctive Treatment and Switching to Aripiprazole on Psychiatric Symptoms}

The aripiprazole and placebo groups did not differ sig-
Table 1. Baseline demographic and clinical data of the subjects

\begin{tabular}{|c|c|c|c|}
\hline \multirow[b]{2}{*}{ Characteristic } & \multicolumn{2}{|c|}{ Group $(n=35)$} & \multirow[b]{2}{*}{$p$ value } \\
\hline & $\begin{array}{l}\text { Placebo } \\
(n=18)\end{array}$ & $\begin{array}{l}\text { Aripiprazole } \\
\qquad(n=17)\end{array}$ & \\
\hline Age (year) & $50.50(2.87)$ & $51.00(2.32)$ & 0.961 \\
\hline Gender (male, \%) & 72.22 & 76.47 & 0.774 \\
\hline $\begin{array}{l}\text { Dose of risperidone } \\
\text { (mg) }\end{array}$ & $3.00(0.30)$ & $3.00(0.37)$ & 0.424 \\
\hline $\begin{array}{l}\text { Duration of illness } \\
\text { (year) }\end{array}$ & $20.00(1.98)$ & $20.00(1.70)$ & 0.732 \\
\hline PANSS & $55.50(3.04)$ & $64.00(3.76)$ & 0.613 \\
\hline Positive & $12.00(0.67)$ & $13.00(1.20)$ & 0.318 \\
\hline Negative & $15.00(1.10)$ & $18.00(1.27)$ & 0.303 \\
\hline General & $27.50(1.63)$ & $27.00(1.63)$ & 0.708 \\
\hline ESRS & $4.50(2.37)$ & $16.00(2.85)$ & 0.153 \\
\hline \multicolumn{4}{|l|}{ Cognitive function } \\
\hline HVLT-Im & $14.00(1.70)$ & $14.00(1.70)$ & 0.883 \\
\hline HVLT-De & $4.50(0.65)$ & $3.00(0.75)$ & 0.503 \\
\hline HVLT-Re & $7.00(0.78)$ & $7.00(0.87)$ & 0.613 \\
\hline Rey & $21.50(1.71)$ & $23.5(1.15)$ & 0.351 \\
\hline Rey-Re & $3.25(0.81)$ & $3.50(1.02)$ & 0.184 \\
\hline Rey-De & $3.75(0.89)$ & $3.5(0.93)$ & 0.909 \\
\hline Rey-Reco & $6.00(0.69)$ & $5.00(0.86)$ & 0.732 \\
\hline DigitSpan-F & $6.00(0.46)$ & $6.00(1.10)$ & 0.757 \\
\hline DigitSpan-B & $3.00(0.41)$ & $3.00(0.61)$ & 0.503 \\
\hline Stroop-L & $112.00(6.33)$ & $112.00(7.02)$ & 0.503 \\
\hline Stroop-C & $60.50(8.48)$ & $60.00(7.88)$ & 0.909 \\
\hline Trail-A & $24.00(0.00)$ & $24.00(0.00)$ & 0.999 \\
\hline Trail-B & $24.00(1.55)$ & $24.00(2.26)$ & 0.152 \\
\hline DSST & $22.00(3.91)$ & $26.00(3.64)$ & 0.757 \\
\hline Verbal & $12.00(0.94)$ & $13.00(1.07)$ & 0.909 \\
\hline Naming & $44.50(2.51)$ & $49.00(2.19)$ & 0.245 \\
\hline Prolactin (ng/ml) & $44.34(8.14)$ & $28.60(10.98)$ & 0.306 \\
\hline
\end{tabular}

Values are presented as median (standard error).

Data were analyzed using the Mann-Whitney test for continuous variables and chi-square analysis for categorical variables.

PANSS, Positive and Negative Syndrome Scale; ESRS, Extrapyramidal Symptom Rating Scale; HVLT-Im, Hopkins verbal learning test free recall (immediately); HVLT-De, Hopkins verbal learning test delayed recall; HVLT-Re, Hopkins verbal learning test recognition; Rey, Rey copy; Rey-Re, Rey immediate recall; Rey-De, Rey delayed recall; Rey-Reco, Rey delayed recognition; DigitSpan-F, digit span forward; DigitSpan-B, digit span backward; Stroop-L, Stroop C-W form letter; Stroop-C, Stroop C-W form color; Trail-A, trail making test part A; Trail-B, trail making test part B; DSST, digit symbol substitution test; Verbal, word fluency; Naming, Korean version of Boston naming test. 
nificantly in terms of changes in total PANSS scores. However, we observed a statistically significant difference in the negative symptoms subscale scores in both

Table 2. Comparison of the results of cognitive function tests

\begin{tabular}{|c|c|c|c|c|}
\hline \multirow{2}{*}{ Item } & \multicolumn{2}{|c|}{ Baseline - Week 12} & \multicolumn{2}{|c|}{ Baseline - Week 24} \\
\hline & $F$ & $p$ value & $F$ & $p$ value \\
\hline HVLT-Im & 1.237 & 0.274 & 1.541 & 0.223 \\
\hline HVLT-De & 0.554 & 0.462 & 1.177 & 0.286 \\
\hline HVLT-Re & 1.633 & 0.210 & 0.158 & 0.693 \\
\hline Rey & 0.057 & 0.813 & 1.914 & 0.176 \\
\hline Rey-Re & 0.004 & 0.950 & 2.877 & 0.099 \\
\hline Rey-De & 1.368 & 0.251 & 0.007 & 0.932 \\
\hline Rey-Reco & 1.293 & 0.264 & 0.595 & 0.446 \\
\hline DigitSpan-F & 0.450 & 0.507 & 0.924 & 0.343 \\
\hline DigitSpan-B & 0.035 & 0.852 & 0.000 & 0.989 \\
\hline Stroop-L & 1.832 & 0.185 & 0.024 & 0.878 \\
\hline Lerr & 1.171 & 0.287 & 1.147 & 0.292 \\
\hline Stroop-C & 2.725 & 0.108 & 3.626 & 0.066 \\
\hline Cerr & 2.306 & 0.138 & 3.632 & 0.065 \\
\hline Trail-A & 1.061 & 0.311 & - & 0.999 \\
\hline Trail-B & 2.660 & 0.112 & 0.418 & 0.522 \\
\hline DSST & 0.010 & 0.922 & 0.987 & 0.328 \\
\hline Verbal & 1.060 & 0.311 & 2.052 & 0.161 \\
\hline Naming & 0.169 & 0.684 & 0.937 & 0.340 \\
\hline
\end{tabular}

Data were analyzed using repeated measures analysis of variance.

HVLT-Im, Hopkins verbal learning test free recall (immediately); HVLT-De, Hopkins verbal learning test delayed recall; HVLT-Re, Hopkins verbal learning test recognition; Rey, Rey copy; Rey-Re, Rey immediate recall; Rey-De, Rey delayed recall; Rey-Reco, Rey delayed recognition; DigitSpan-F, digit span forward; DigitSpan-B, digit span backward; Stroop-L, Stroop C-W form letter; Stroop-C Stroop C-W form color: Trail-A, trail making test part A; Trail-B, trail making test part B: DSST, digit symbol substitution test: Verbal, word fluency; Naming, Korean version of Boston naming test. phases of the study $(p<0.05$; Table 3$)$.

\section{Effect of Adjunctive Treatment and Switching to Aripiprazole on Serum Prolactin}

Serum prolactin levels decreased significantly over 24 weeks in the aripiprazole group (baseline, $40.18 \pm 10.98$ $\mathrm{ng} / \mathrm{ml} ; 24$ weeks, $20.35 \pm 13.81 \mathrm{ng} / \mathrm{ml} ; p<0.001)$. In contrast, the mean serum prolactin levels did not change significantly in the placebo group (baseline, 50.40 \pm 8.14 $\mathrm{ng} / \mathrm{ml} ; 24$ weeks, $45.07 \pm 7.23 \mathrm{ng} / \mathrm{ml} ; p=0.34)$. It is noteworthy that the serum prolactin levels in the aripiprazole group decreased significantly within the first week of treatment $(p<0.05)$. The repeated-measures ANOVA of

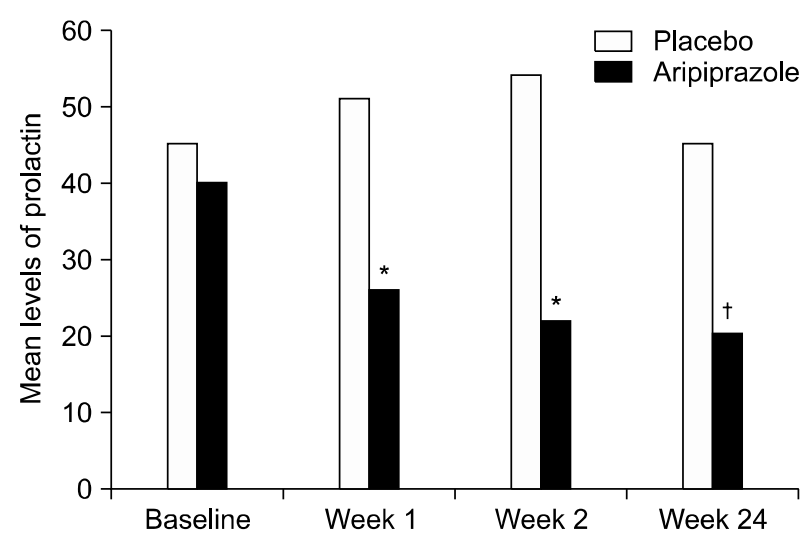

Fig. 2. Mean change from baseline to week 24 in serum prolactin levels.

${ }^{*} \mathrm{p}<0.05,{ }^{\dagger} \mathrm{p}<0.001$

Table 3. Repeated measures ANOVA comparing placebo and aripiprazole group in serum prolactin levels

\begin{tabular}{|c|c|c|c|c|c|c|c|c|}
\hline \multicolumn{3}{|c|}{ Between-group differences (group effect) } & \multicolumn{3}{|c|}{ Within-group differences (time effect) } & \multicolumn{3}{|c|}{ Group×time interaction } \\
\hline $\mathrm{F}$ & d.f. & $p$ value & $\mathrm{F}$ & d.f. & $p$ value & $\mathrm{F}$ & d.f. & $p$ value \\
\hline 2.59 & 1 & 0.12 & 4.85 & 2 & 0.01 & 9.25 & 2 & 0.00 \\
\hline
\end{tabular}

ANOVA, analysis of variance; d.f., degree of freedom.

Table 4. Results of assessment of psychopathology and side-effects parameters

\begin{tabular}{|c|c|c|c|c|}
\hline & Items & Baseline & Week 12 & Week 24 \\
\hline \multirow[t]{5}{*}{ Placebo $(n=18)$} & PANSS total & $56.72(3.04)$ & $53.78(3.04)$ & 53.61 (3.09) \\
\hline & Positive & $12.50(0.67)$ & $12.33(0.70)$ & $12.44(0.76)$ \\
\hline & Negative & $15.39(1.10)$ & $15.17(1.08)$ & 15.39 (1.09) \\
\hline & General & $28.83(1.63)$ & $26.83(1.61)$ & $26.33(1.60)$ \\
\hline & ESRS & $9.28(2.37)$ & $8.67(2.29)$ & $8.22(2.13)$ \\
\hline \multirow[t]{5}{*}{ Aripiprazole $(n=17)$} & PANSS total & $59.53(3.76)$ & $54.00(2.92)$ & $52.47(3.44)$ \\
\hline & Positive & $14.47(1.20)$ & $12.47(0.88)$ & $12.65(0.93)$ \\
\hline & Negative & $17.24(1.27)$ & $14.71(0.97)$ & $14.71(1.25)$ \\
\hline & General & $26.24(1.45)$ & $25.12(1.50)$ & $25.12(1.66)$ \\
\hline & ESRS & $14.53(2.85)$ & $8.53(2.36)$ & $7.00(1.84)$ \\
\hline
\end{tabular}

Values are presented as mean (standard error).

PANSS. Postive and Negative Syndrome Scale; ESRS, Extrpyramidal Syndrome Rating Scale. 
the log-transformed serum prolactin levels revealed significant changes in prolactin levels in the aripiprazole group during the trial period ( $p<0.05$; Table 3 ). Moreover, the between-group difference in serum prolactin level from baseline to endpoint (week 24) was significant $(p<0.05$; Fig. 2).

\section{Effect of Adjunctive Treatment and Switching on EPS}

No change in the dose or frequency of anticholinergic drugs occurred during the study period. We found between-group differences in ESRS scores in both phases of the study $(p<0.05)$. In the aripiprazole group, the mean ESRS score at baseline decreased during the first phase and remained stable to the study endpoint (Table 4).

\section{Adverse Events Associated with the Adjunctive Treatment and Switch to Aripiprazole}

Three subjects dropped out of the study because of symptom aggravation (two patients in the aripiprazole group and one patient in the placebo group). With the exception of those three subjects, no serious adverse events caused a subject to withdraw from the study. About $70 \%$ of subjects reported no treatment-emergent adverse events following the addition of aripiprazole to their regimen, and the rest reported only mild and tolerable adverse events. Three patients in the aripiprazole group complained of an upper respiratory tract infection (URI), URI-like symptoms, irritability, and headache. Four patients in the placebo group complained of URI-like symptoms.

\section{DISCUSSION}

In the present multicenter, randomized, double-blind, placebo-controlled, open-label trial, adjunctive treatment and switching were not associated with improvement in the cognitive impairments exerperienced by patients with schizophrenia receiving a stable dose of risperidone. Furthermore, with the exception of negative symptoms, adjunctive aripiprazole was not associated with a change in schizophrenia symptoms. Switching to aripiprazole and adjunctive strategies significantly reduced risperidone-induced EPS. This result may be attributable to the unique properties of aripiprazole that enable stabilization of the dopamine system via competition with risperidone.

Importantly, adjunctive aripiprazole reversed hyperprolactinemia induced by risperidone within 1 week. This finding supports the previously postulated role for aripiprazole in alleviating antipsychotic-induced hyperprolac- tinemia. ${ }^{22,27-30)}$ To our knowledge, the present study is first report of adjunctive aripiprazole decreasing risperidone-induced serum prolactin levels within 1 week of administration. Previous studies have shown changes in prolactin levels after 2 weeks. ${ }^{22,27-30)}$ Byerly et al. ${ }^{28)}$ reported a decrease in prolactin 1 week after aripiprazole administration; however, their strategy of simultaneously tapering the concomitant antipsychotics and administering aripiprazole may have contributed to the decrease in serum prolactin levels. A low dose of aripiprazole, such as $10 \mathrm{mg} /$ day, can reduce prolactin levels without adverse effects. Mace and Taylor ${ }^{31)}$ reported that aripiprazole exhibited the highest affinity for the $\mathrm{D}_{2}$ receptors at 10 $\mathrm{mg} /$ day.

Aripiprazole has a higher affinity for $\mathrm{D}_{2}$ receptors than does risperidone ${ }^{22)}$ Adjunctive treatment with aripiprazole in patients receiving risperidone may optimize $\mathrm{D}_{2}$-receptor activity and, hence, diminish the risk of EPS associated with risperidone. Furthermore, adjunctive treatment decreases the elevation in prolactin levels resulting from the high $\mathrm{D}_{2}$-receptor occupancy by the full antagonist. ${ }^{22)}$

There are several possible explanations for why our study failed to show a beneficial effect of aripiprazole on the cognitive impairment of patients with chronic schizophrenia. First, the patients in our trial were hospitalized and had had a long duration of illness. Second, our sample size was small. Despite the stabilizing dose of risperidone, most of our subjects had prolonged hospital stays and a mean duration of illness of approximately 17 years.

Several observational studies have shown that aripiprazole improves cognitive impairment in patients with schizophrenia. ${ }^{17-21)}$ However, few pragmatic randomized trials have investigated the clinical effect of aripiprazole on cognitive impairment in schizophrenia. Two such studies stand out. In a study by Kane and colleagues, ${ }^{22)} 323$ outpatients randomly received aripiprazole or placebo as an adjunct to a stable regimen of quetiapine or risperidone. This double-blind comparison did not reveal improvement in psychiatric symptoms, including cognitive impairment, after 16 weeks of treatment with aripiprazole. Benefits in terms of decreased prolactin or triglycerides were weighed against the risk of metabolic problems. However, Kane et al. ${ }^{22)}$ did not report on the duration of illness of their subjects. Yasui-Furukori et al. ${ }^{23)}$ conducted a 12-week double-blind trial in which 36 outpatients with schizophrenia who were receiving risperidone or olanzapine were randomly treated with aripiprazole or placebo. Despite a worsening in some domains of cognitive func- 
tion, adjunctive aripiprazole treatment improved motor speed. Although no statistically significant betweengroup differences in illness duration were observed, the average illness duration of the aripiprazole group was about 7 years.

Overall, the observational studies have shown that aripiprazole improved cognitive function in patients with schizophrenia. However, the majority of subjects in these studies were first-episode patients or their duration of illness did not exceed 10 years. Furthermore, the subjects in the majority of studies that reported positive findings were outpatients. Chronic patients with schizophrenia present with more generalized cognitive decline than do first-episode patients, and cognitive function declines over the course of the illness. ${ }^{32-34)}$ The 24-week study period in the present study may not have been long enough to reveal an effect of aripiprazole on the cognitive impairment of patients with schizophrenia. Negative symptoms significantly influence and predict cognitive function in schizophrenia. $^{20)}$ In the present study, negative symptoms improved after adjunctive treatment with or switch to aripiprazole. This finding suggests that additional randomized, long-term studies with a large sample would be useful for determining the role of aripiprazole in the management of cognitive impairment in patients with schizophrenia.

The present study had two phases: a 12-week doubleblind, placebo-controlled, randomized phase that evaluated the effect of administering aripiprazole as an adjunct to risperidone, and a 12-week open-label phase to confirm the stability and long-term efficacy of the switch to aripiprazole after 12 weeks of combination treatment. Despite a negative finding on the primary outcome measure, we propose that switching after combining two atypical antipsychotics with different properties is a potential treatment strategy for schizophrenia characterized by intractable, disabling problems.

Despite its use of a rigorous design, which included a double-blind, placebo-controlled, randomized, and open-label follow-up approach, the present study has several limitations. First, the number of subjects was small $(n=35)$. Second, few female subjects were included in the study (four in the aripiprazole group and five in the placebo group), and we did not distinguish between males and females in the data analysis. However, a separate reanalysis of data from only female subjects revealed no statistically significant differences between aripiprazole and placebo groups. This negative finding may be the result of the small sample size. Fourth, methodological differences be- tween the second phase of our study and previous adjunctive aripiprazole studies prohibit direct comparisons. Finally, it is recommended that plasma concentrations of risperidone or aripiprazole be checked to monitor drug interactions. $^{35)}$

In conclusion, administering aripiprazole as an adjunct to risperidone and subsequently switching to aripiprazole did not significantly improve the cognitive function of patients with schizophrenia. However, adjunctive aripiprazole therapy was generally well tolerated and may be beneficial for reducing negative symptoms, partially by decreasing extrapyramidal side effects, and for reducing hyperprolactinemia induced by previous antipsychotic drugs. Further studies to evaluate the efficacy of adjunctive aripiprazole therapy are warranted.

\section{REFERENCES}

1. Green MF. Cognitive impairment and functional outcome in schizophrenia and bipolar disorder. J Clin Psychiatry 2006; 67:e12.

2. Lee BJ, Lee JG, Kim YH. A 12-week, double-blind, placebo-controlled trial of donepezil as an adjunct to haloperidol for treating cognitive impairments in patients with chronic schizophrenia. J Psychopharmacol 2007;21:421-427.

3. Lee SW, Lee JG, Lee BJ, Kim YH. A 12-week, double-blind, placebo-controlled trial of galantamine adjunctive treatment to conventional antipsychotics for the cognitive impairments in chronic schizophrenia. Int Clin Psychopharmacol 2007;22:63-68.

4. Lee JG, Lee SW, Lee BJ, Park SW, Kim GM, Kim YH. Adjunctive memantine therapy for cognitive impairment in chronic schizophrenia: a placebo-controlled pilot study. Psychiatry Investig 2012;9:166-173.

5. Shim JC, Jung DU, Jung SS, Seo YS, Cho DM, Lee JH, et al. Adjunctive varenicline treatment with antipsychotic medications for cognitive impairments in people with schizophrenia: a randomized double-blind placebo-controlled trial. Neuropsychopharmacology 2012;37:660-668.

6. Bilder RM, Goldman RS, Volavka J, Czobor P, Hoptman M, Sheitman B, et al. Neurocognitive effects of clozapine, olanzapine, risperidone, and haloperidol in patients with chronic schizophrenia or schizoaffective disorder. Am J Psychiatry 2002;159:1018-1028.

7. Woodward ND, Purdon SE, Meltzer HY, Zald DH. A metaanalysis of neuropsychological change to clozapine, olanzapine, quetiapine, and risperidone in schizophrenia. Int $J$ Neuropsychopharmacol 2005;8:457-472.

8. Byerly M, Suppes T, Tran QV, Baker RA. Clinical implications of antipsychotic-induced hyperprolactinemia in patients with schizophrenia spectrum or bipolar spectrum disorders: recent developments and current perspectives. $J$ Clin Psychopharmacol 2007;27:639-661.

9. Bostwick JR, Guthrie SK, Ellingrod VL. Antipsychoticinduced hyperprolactinemia. Pharmacotherapy 2009;29:6473.

10. Burris KD, Molski TF, Xu C, Ryan E, Tottori K, Kikuchi $\mathrm{T}$, et al. Aripiprazole, a novel antipsychotic, is a highaffinity partial agonist at human dopamine D2 receptors. $J$ 
Pharmacol Exp Ther 2002;302:381-389.

11. Tadori Y, Forbes RA, McQuade RD, Kikuchi T. Characterization of aripiprazole partial agonist activity at human dopamine D3 receptors. Eur J Pharmacol 2008;597:27-33.

12. Jordan S, Koprivica V, Chen R, Tottori K, Kikuchi T, Altar CA. The antipsychotic aripiprazole is a potent, partial agonist at the human 5-HTIA receptor. Eur J Pharmacol 2002; 441:137-140.

13. Hirose $\mathrm{T}$, Uwahodo $\mathrm{Y}$, Yamada $\mathrm{S}$, Miwa $\mathrm{T}$, Kikuchi $\mathrm{T}$, Kitagawa $\mathrm{H}$, et al. Mechanism of action of aripiprazole predicts clinical efficacy and a favourable side-effect profile. J Psychopharmacol 2004;18:375-383.

14. Jordan S, Koprivica V, Dunn R, Tottori K, Kikuchi T, Altar CA. In vivo effects of aripiprazole on cortical and striatal dopaminergic and serotonergic function. Eur J Pharmacol 2004;483:45-53.

15. Kwon JS, Jang JH, Kang DH, Yoo SY, Kim YK, Cho SJ; APLUS Study Group. Long-term efficacy and safety of aripiprazole in patients with schizophrenia, schizophreniform disorder, or schizoaffective disorder: 26-week prospective study. Psychiatry Clin Neurosci 2009;63:73-81.

16. Li Z, Ichikawa J, Dai J, Meltzer HY. Aripiprazole, a novel antipsychotic drug, preferentially increases dopamine release in the prefrontal cortex and hippocampus in rat brain. Eur J Pharmacol 2004;493:75-83.

17. Mucci A, Piegari G, Galderisi S. Cognitive-enhancing effects of aripiprazole: a case report. Clin Pract Epidemiol Ment Health 2008;4:24.

18. Kim SW, Shin IS, Kim JM, Lee JH, Lee YH, Yang SJ, et al. Effectiveness of switching to aripiprazole from atypical antipsychotics in patients with schizophrenia. Clin Neuropharmacol 2009;32:243-249.

19. Riedel M, Spellmann I, Schennach-Wolff R, Musil R, Dehning S, Cerovecki A, et al. Effect of aripiprazole on cognition in the treatment of patients with schizophrenia. Pharmacopsychiatry 2010;43:50-57.

20. Riedel M, Schennach-Wolff R, Musil R, Dehning S, Cerovecki A, Opgen-Rhein $\mathrm{M}$, et al. Neurocognition and its influencing factors in the treatment of schizophrenia-effects of aripiprazole, olanzapine, quetiapine and risperidone. Hum Psychopharmacol 2010;25:116-125.

21. Suzuki H, Gen K, Inoue Y. An unblinded comparison of the clinical and cognitive effects of switching from first-generation antipsychotics to aripiprazole, perospirone or olanzapine in patients with chronic schizophrenia. Prog Neuropsychopharmacol Biol Psychiatry 2011;35:161-168.

22. Kane JM, Correll CU, Goff DC, Kirkpatrick B, Marder SR, Vester-Blokland $\mathrm{E}$, et al. A multicenter, randomized, doubleblind, placebo-controlled, 16-week study of adjunctive aripiprazole for schizophrenia or schizoaffective disorder inadequately treated with quetiapine or risperidone monotherapy. J Clin Psychiatry 2009;70:1348-1357.
23. Yasui-Furukori N, Kaneda A, Sugawara N, Tomita T, Kaneko S. Effect of adjunctive treatment with aripiprazole to atypical antipsychotics on cognitive function in schizophrenia patients. J Psychopharmacol 2012;26:806-812.

24. Shabsigh R, Rowland D. The Diagnostic and Statistical Manual of Mental Disorders, fourth edition, text revision as an appropriate diagnostic for premature ejaculation. J Sex Med 2007;4:1468-1478.

25. Kay SR, Fiszbein A, Opler LA. The positive and negative syndrome scale (PANSS) for schizophrenia. Schizophr Bull 1987;13:261-276.

26. Chouinard G, Margolese HC. Manual for the Extrapyramidal Symptom Rating Scale (ESRS). Schizophr Res 2005;76:247265.

27. Shim JC, Shin JG, Kelly DL, Jung DU, Seo YS, Liu KH, et al. Adjunctive treatment with a dopamine partial agonist, aripiprazole, for antipsychotic-induced hyperprolactinemia: a placebo-controlled trial. Am J Psychiatry 2007;164:14041410.

28. Byerly MJ, Marcus RN, Tran QV, Eudicone JM, Whitehead $\mathrm{R}$, Baker RA. Effects of aripiprazole on prolactin levels in subjects with schizophrenia during cross-titration with risperidone or olanzapine: analysis of a randomized, open-label study. Schizophr Res 2009;107:218-222.

29. Chen CK, Huang YS, Ree SC, Hsiao CC. Differential add-on effects of aripiprazole in resolving hyperprolactinemia induced by risperidone in comparison to benzamide antipsychotics. Prog Neuropsychopharmacol Biol Psychiatry 2010; 34:1495-1499.

30. Yasui-Furukori N, Furukori H, Sugawara N, Fujii A, Kaneko S. Dose-dependent effects of adjunctive treatment with aripiprazole on hyperprolactinemia induced by risperidone in female patients with schizophrenia. J Clin Psychopharmacol 2010;30:596-599.

31. Mace S, Taylor D. Aripiprazole: dose-response relationship in schizophrenia and schizoaffective disorder. CNS Drugs 2009;23:773-780.

32. Agius M, Goh C, Ulhaq S, McGorry P. The staging model in schizophrenia, and its clinical implications. Psychiatr Danub 2010;22:211-220.

33. Lewandowski KE, Cohen BM, Ongur D. Evolution of neuropsychological dysfunction during the course of schizophrenia and bipolar disorder. Psychol Med 2011;41:225-241.

34. Ojeda N, Peña J, Schretlen DJ, Sánchez P, Aretouli E, Elizagárate $\mathrm{E}$, et al. Hierarchical structure of the cognitive processes in schizophrenia: the fundamental role of processing speed. Schizophr Res 2012;135:72-78.

35. Urichuk L, Prior TI, Dursun S, Baker G. Metabolism of atypical antipsychotics: involvement of cytochrome p450 enzymes and relevance for drug-drug interactions. Curr Drug Metab 2008;9:410-418. 